UCRL-JC-124350

PREPRINT

$$
\text { CONF- } 9607129-1
$$

\title{
Model-Based Signal Processing for Laser Ultrasonic Signal Enhancement
}
R. D. Huber
D. J. Chinn
G. H. Thomas
J. V. Candy
J. B. Spicer

RECEIVED

SEP 201996

OSTI

This paper was prepared for submittal to the

23rd Annual Review of Progress in Quantitative Nondestructive Evaluation

Brunswick, Maine

July 28-August 2, 1996

July 1996

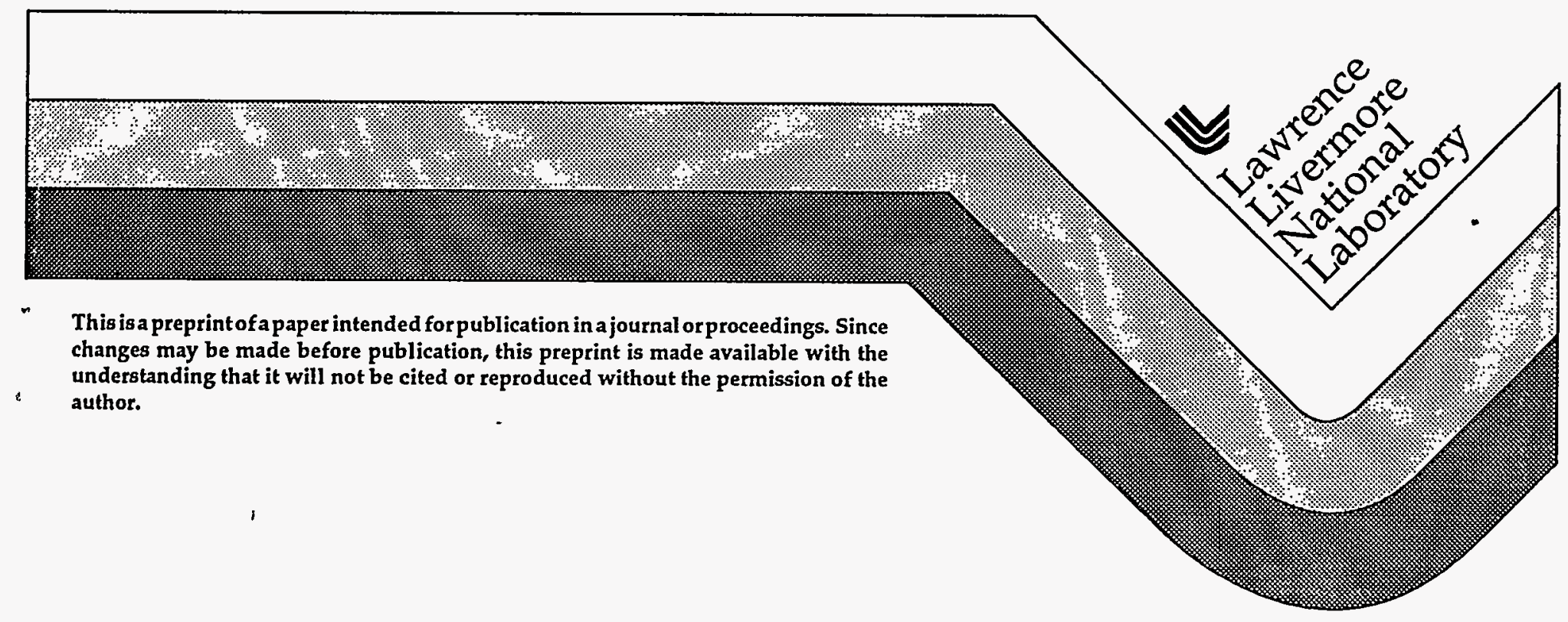




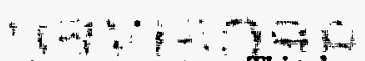

DISCLAIMER

This document was prepared as an account of work sponsored by an agency of the United States Government. Neither the United States Covernment nor the University of California nor any of their employees, makes any warranty, express or implied, or assumes any legal liability or responsibility for the accuracy, completeness, or usefulness of any information, apparatus, product, or process disclosed, or represents that its use would not infringe privately owned rights. Reference herein to any specific commercial product, process, or service by trade name, trademark, manufacturer, or otherwise, does not necessarily constitute or imply its endorsement, recommendation, or favoring by the United States Government or the University of California. The views and opinions of authors expressed herein do not necessarily state or reflect those of the United States Government or the University of California, and shall not be used for advertising or product endorsement purposes. 


\section{DISCLAIMER}

Portions of this document may be illegible in electronic image products. Images are produced from the best available original document. 
MODEL-BASED SIGNAL PROCESSING FOR LASER

\title{
ULTRASONIC SIGNAL ENHANCEMENT
}

\author{
R. D. HUBER D. J. CHINN G. H. THOMAS J. V. CANDY \\ Lawrence Livermore National Laboratory \\ P.O. Box 808, L-228 \\ Livermore, CA 94551
}

\section{J. B. SPICER}

The Johns Hopkins University

Center for Nondestructive Evaluation

Baltimore, MD 21218

\section{INTRODUCTION}

The use of laser-based ultrasonics in the testing of materials and structures offers various advantages over more traditional ultrasonic methods, but is often less sensitive when applied to real materials. Although high energy laser pulses can generate large ultrasonic displacements, nondestructive evaluation requires that the ablation regime be avoided, thus limiting the amount of optical energy which may be used. For this reason, signal processing of laser generated ultrasonic waveforms detected using laser interferometers may be required to extract the desired information from a nondestructive laser ultrasonic test. A model-based signal processing technique offers a way to enhance the signal-to-noise ratios significantly for ultrasonic waveforms obtained using laser-based systems with the generation of the ultrasound occurring in the nondestructive thermoelastic regime.

Under ideal conditions, good signal-to-noise ratios can be achieved using laser-based ultrasonics. However, many materials which need to be tested have less than ideal surface finishes for optical detectors. The application of signal processing to laser-based ultrasonics may provide the necessary improvement in sensitivity. Aussel and Monchalin used cross-correlation methods to extract acoustic velocities and elastic constants from noisy measurements [1]. Once the constants are made available through experimentation or calculation, it is possible to enhance the noisy interferometer measurements even further by generating a predicted or reference response using a propagation model that captures the essence of the displacement signal to be estimated. Using estimates of the required constants, a reasonable reference response can be generated that enables significant enhancement of the measured displacement. This work uses a model-reference approach to increase the signal-to-noise ratio in noisy laser-based ultrasonic waveforms.

\section{LASER ULTRASONIC PROPAGATION MODELING}

A model for calculating the surface displacement at any point on the surface of a plate resulting from an incident pulse of laser energy has been developed by Spicer [2]. The model calculates the surface displacements resulting from a thermoelastic source. The equations (in cylindrical coordinates) governing the laser ultrasonic waves in the plate are given in terms of scalar 


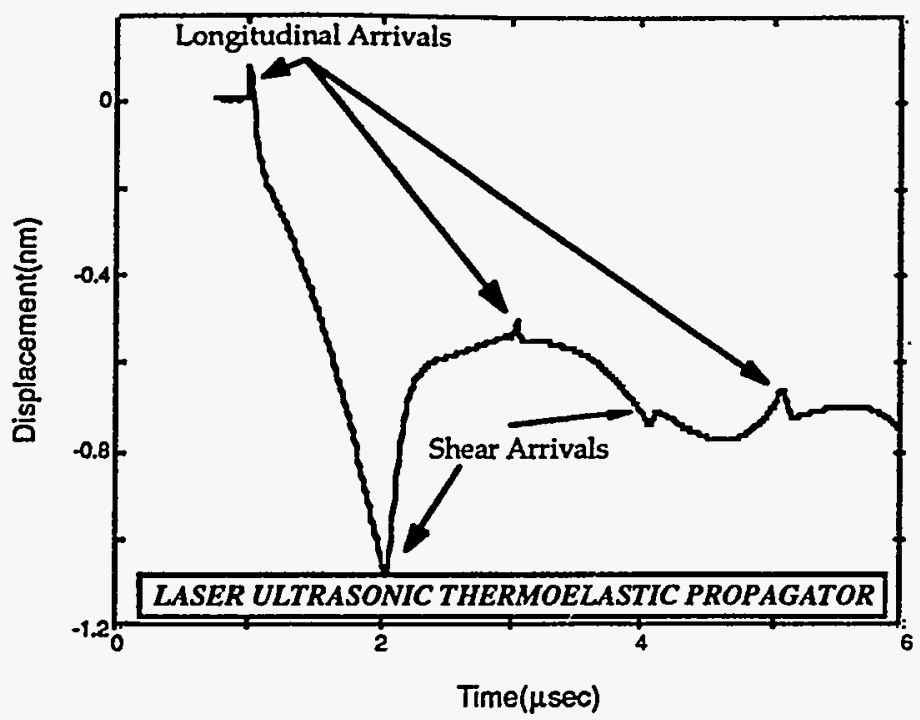

Figure 1: Synthesized Surface Displacement: 6mm Aluminum Plate.

and vector potentials defined as $\phi(r, z, t), \psi(r, z, t)$, respectively. The vector surface displacement, $\mathrm{d}(r, z, t)$ is then constructed in terms of these potentials as:

$$
\mathrm{d}(r, z, t)=\nabla \phi(r, z, t)+\nabla \times \psi(r, z, t)
$$

Calculations are performed in the frequency domain, and the final surface displacement is found by inverting the Hankel-Laplace representation in complex variables $\mathrm{p}$ and $\mathrm{s}$ :

$$
d(p, z, s)=\Phi(p, z, s)+p^{2} \psi(p, z, s)
$$

that is, the surface displacement is:

$$
d(r, z, t)=\mathrm{H}_{0}^{-1}\left[\mathrm{~L}^{-1}[d(p, z, s)]\right] .
$$

The simulation algorithm solves these equations to produce the response for a given material, thickness, etc. Figure 1 shows the calculated waveform on epicenter on the side of the plate opposite that on which generation took place for a $6 \mathrm{~mm}$-thick aluminum plate using the constants associated with isotropic material behavior. The following constants were used in the calculation: longitudinal wave speed, $6.32 \mathrm{~mm} / \mu s$; shear wave speed, $3.11 \mathrm{~mm} / \mu s$; thermal conductivity, $0.235 \mathrm{~W} / \mathrm{mm} \mathrm{K}$; thermal diffusivity, $9.67 \times 10^{-5} \mathrm{~mm}^{2} / \mu s$; and linear thermal expansion coefficient, $2.4 \times 10^{-5}{ }^{\circ} \mathrm{C}^{-1}$; along with the laser parameters obtained from manufacturer specifications. Figure 1 shows the typical surface displacement response which includes the initital longitudinal and shear arrivals, as well as longitudinal and shear arrivals corresponiding to multiple traversals through the plate.

\section{MODEL-REFERENCE LASER ULTRASONIC PROCESSING}

For the experimentally obtained waveforms found in this work, a Michelson interferometer was used to measure the ultrasonic surface displacement. For a perfectly adjusted instrument and "small" surface displacements, the measured intensity is directly proportional to the displacement, that is,

$$
I(z, t) \propto \alpha d(t)
$$

where $\alpha$ is a constant that is a function of wave number, photodetector efficiency, carrier charge, frequency of the light and required scale factors. 
There are many sources of noise in interferometric systems including detector quantum noise and dark current, generation-recombination noise, thermal noise from the electronics, laser noise. All of these noise sources are grouped together and considered simply a measurement noise term to give the measurement system model as

$$
x\left(t_{k}\right)=I\left(z_{\ell}, t_{k}\right)+n\left(t_{k}\right)=\alpha d\left(t_{k}\right)+n\left(t_{k}\right)
$$

with $x\left(t_{k}\right)$ the measured surface displacement at time sample $t_{k}, d\left(t_{k}\right)$ the actual or true displacement and $n\left(t_{k}\right)$ the lumped random noise contaminating the process.

Ideally, one would like to construct a processor that linearly combines the raw set of sampled (digitized) measurements $\left\{x\left(t_{k}\right)\right\}, k=0, \cdots, N$ to produce an optimal estimate of the desired displacement, that is,

$$
\hat{d}\left(t_{k}\right)=\sum_{\ell=0}^{L-1} w_{\ell} x\left(t_{k}-t_{\ell}\right)=\mathbf{w}^{T} \mathbf{X}\left(t_{k}\right),
$$

where $\mathbf{w}^{T}=\left[w_{0} \cdots w_{L-1}\right]$ and $\mathrm{X}^{T}=\left[x\left(t_{k}\right) \cdots x\left(t_{k}-t_{L-1}\right)\right]$.

One approach to solve this problem is to formulate it as a minimum variance estimation problem:

GIVEN a set of noisy interferometric measurements, $\left\{x\left(t_{k}\right)\right\}, k=0, \cdots, N$; FIND the best (minimum error variance) estimate of the surface displacement as $\hat{d}\left(t_{k}\right)$.

Mathematically, the set of weights that minimizes the well-known mean-squared error criterion must be found. The solution to this problem is classical [3] and is easily found by differentiating $J$ with respect to the weights, setting the result equal to zero and solving for the set of optimal weights which gives the so-called normal equations,

$$
\mathbf{w}_{\text {opt }}=\mathbf{R}_{x x}^{-1} \mathbf{R}_{d x}
$$

where $\mathbf{R}_{x x}$ is a Toeplitz correlation matrix of the data and $\mathbf{R}_{d x}$ is the cross-correlation vector between the measurements and desired signal (model reference). The corresponding minimum mean-squared error is easily determined as

$$
J_{\min }=\sigma_{d}^{2}-\mathbf{R}_{d x}^{T} \mathbf{w}_{o p t},
$$

with $\sigma_{d}^{2}$ the variance of the reference response. An efficient approach to solve for the optimal weights is obtained using the so-called Levinson-Wiggins-Robinson (LWR) recursion which recursively solves a slight variant of the above relation given by

$$
\mathbf{w}_{\text {opt }}(L)=\left(\mathbf{R}_{x x}(L)+\sigma_{n}^{2} \mathbf{I}\right)^{-1} \mathbf{R}_{d x}(L)
$$

where the two available "tuning" parameters are $L$, the filter order, and $\sigma_{n}^{2}$ the regularization parameter which can shown to be proportional to the measurement noise variance. The performance of the algorithm can be determined by tuning with a particular set of parameters, $\left[L, \sigma_{n}^{2}\right]$ and calculating the resulting mean-squared error, $J_{\min }$-the parameter set yielding the smallest mean-squared error is the best choice.

Another alternative approach to solving the normal equations is by developing an equivalent adaptive solution using the well-known least mean-squared (LMS) algorithm [3]. The advantage of this approach is computational in that it is proportional to $L$-operations to perform the required 


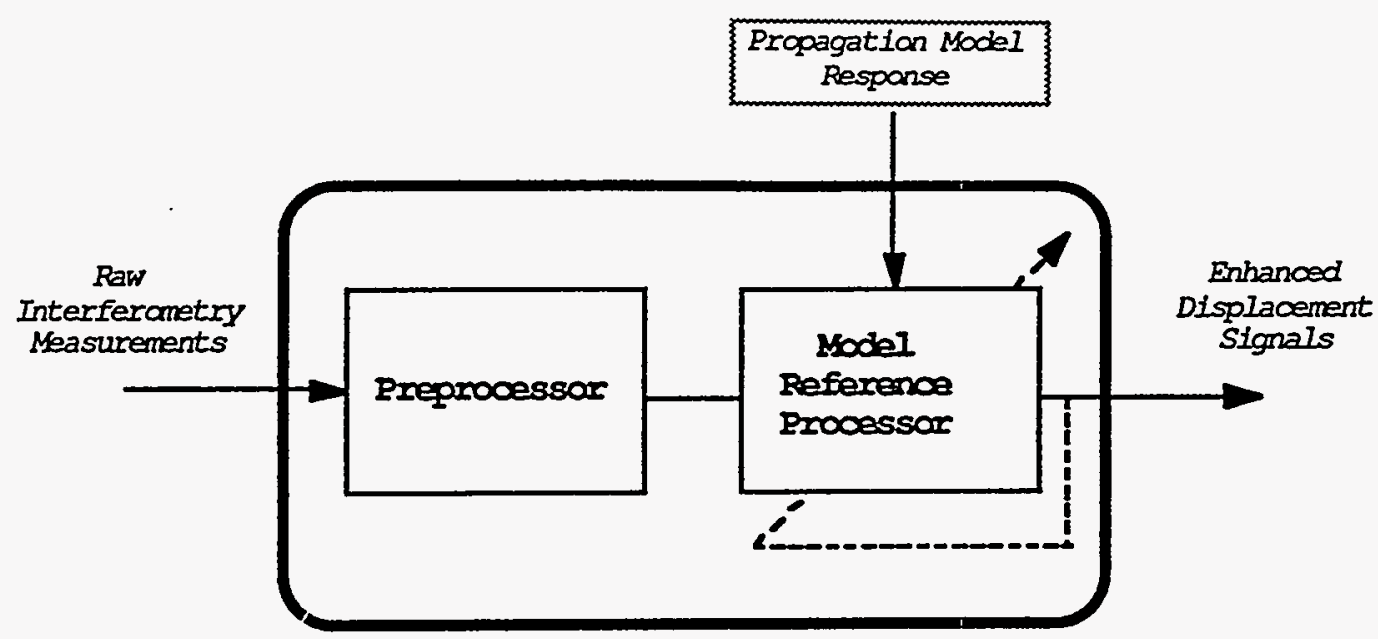

Figure 2: Model-Reference Processor Structure: Optimal and Adaptive (dashed lines).

inverse $\left(\mathbf{R}_{x x}^{-1}\right)$ instead of the $L^{2}$-operations required by the LWR algorithm. Also in this approach the instantaneous gradient replaces the stochastic gradient, [3] allowing for the inclusion of non-stationary processes, since it is time recursive. The LMS recursion for updating the weights is given by

$$
\mathbf{w}\left(t_{k+1}\right)=\mathbf{w}\left(t_{k}\right)+\Delta \epsilon\left(t_{k}\right) \mathbf{X}\left(t_{k}\right)
$$

where the instantaneous error is given by

$$
\epsilon\left(t_{k}\right)=d\left(t_{k}\right)-\hat{d}\left(t_{k}\right)=d\left(t_{k}\right)-\mathbf{w}^{T}\left(t_{k}\right) \mathbf{X}\left(t_{k}\right),
$$

and $\Delta$ is the gradient (search) step-size which (for convergence) is bounded by 0 and $2 / \lambda_{\max }$, with $\lambda_{\max }$ the maximum eigenvalue of $\mathbf{R}_{x x}$. It should also be noted that for stationary processes the adaptive algorithm will converge precisely to the optimal solution, that is, $w\left(t_{k}\right) \longrightarrow \mathbf{w}_{\text {opt }}$. As before, the parameters to tune the LMS algorithm are $L$ the filter order, $\Delta$ the step-size parameter and $N_{i}$ the number of iterations through the data set, where the $i^{\text {th }}$-iteration of the weight recursion becomes

$$
\mathrm{w}_{i}\left(t_{k+1}\right)=\cdot \mathrm{w}_{i}\left(t_{k}\right)+\triangle_{i} \epsilon\left(t_{k}\right) \mathbf{X}\left(t_{k}\right)
$$

The generic structure for the model-reference processor is shown in Figure 2. After pre-processing the raw interferometric data with a low-pass filter the optimal or adaptive surface displacement estimate is obtained using either the LWR or adaptive LMS algorithm. Figure 3 shows the application of the model-reference processor to some experimental data. In this figure, the desired or model reference response predicted by the propagation model for the particular material along with the corresponding optimal (upper trace) and adaptive (lower trace) surface displacement estimates is shown. In this case it is clear that both implementations of the processor perform quite well in extracting and enhancing the signal. For a "real-time" implementation, the adaptive processor would be the choice due to its lighter computational load and the fact that it can be tuned to near optimal performance. Next, both algorithms are applied to various sets of experimental data.

\section{LASER ULTRASONICS EXPERIMENT}

All of the signals recorded using the Michelson interferometer were taken on epicenter in a $6 \mathrm{~mm}$ thick, aluminum alloy plate. Incident laser pulse energy was varied from a maximum of $35 \mathrm{~mJ}$. The temporal origin of the signals is taken at the instant (time $=0$ ) of laser firing. Thus, prior to firing the initial portion of the data is a record of the system and background noise before the arrival of the laser pulse at the specimen surface. After the firing, electronic noise contaminates the laser 


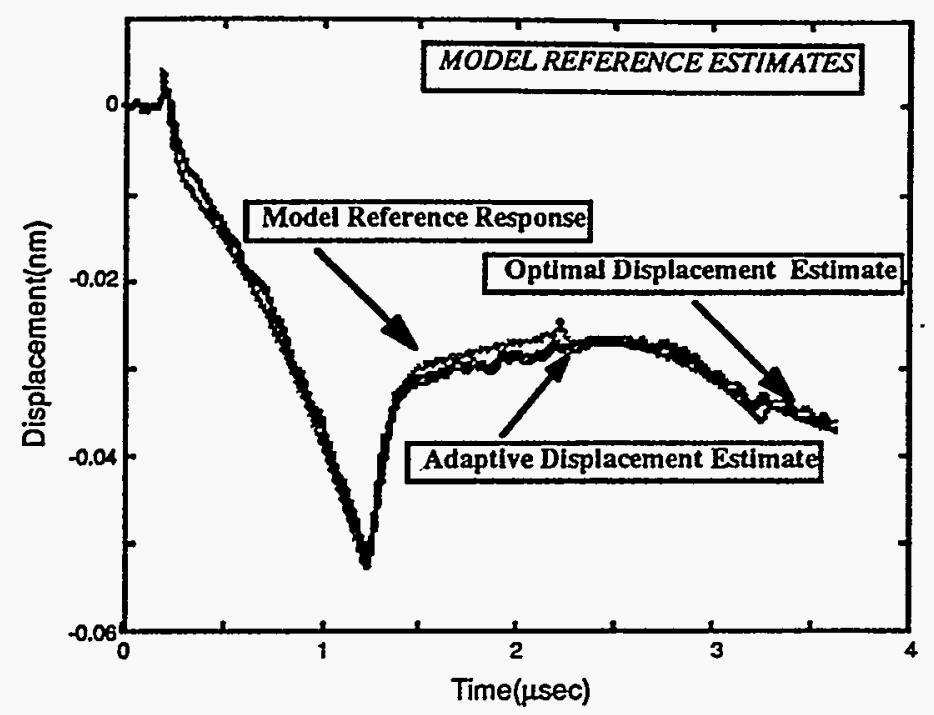

Figure 3: Typical Enhanced Surface Displacement Estimate: Model Reference, Estimate, and Adaptive Estimate (28dB SNR).

generated ultrasonic signal before the arrival of the longitudinal wave. This noise is a problem in that it can continue for hundreds of nanoseconds interfering with the detection of the ultrasonic signal. A typical measurement is shown in Figure 4.

The following set of signal-to-noise ratios are defined to quantify the performance of the processors. On input, the average power in the model-reference signal is defined by

$$
P_{s} \equiv \frac{1}{T} \int_{0}^{T} d^{2}(t) d t \approx \frac{1}{N} \sum_{k=0}^{N} d^{2}\left(t_{k}\right),
$$

and the variance of the noise, $\sigma_{n}^{2}$, is used to calculate the input SNR

$$
S N R_{i n} \equiv \frac{P_{s}}{\sigma_{n}^{2}}
$$

The noise variance is estimated by applying a 100-sample window averaging filter to the measured signal, subtracting this averaged signal and calculating the sample variance

$$
\sigma_{n}^{2}=\frac{1}{N} \sum_{k=0}^{N}\left(x\left(t_{k}\right)-\bar{x}\left(t_{k}\right)\right)^{2} .
$$

The output SNR uses the same signal average power, but the noise variance in this case is the estimation error variance with the bias removed, since it represents the residual remaining after model-reference processing, that is, with the error defined as before with $e\left(t_{k}\right)=d\left(t_{k}\right)-\hat{d}\left(t_{k}\right)$, we have

$$
\sigma_{e}^{2}=\frac{1}{N} \sum_{k=0}^{N}\left(e\left(t_{k}\right)-\bar{e}\left(t_{k}\right)\right)^{2},
$$

giving

$$
S N R_{\text {out }} \equiv \frac{P_{s}}{\sigma_{e}^{2}}
$$

When applying this approach to the experimental data, the procedure consists of: (1) preprocessing using a 10-sample window averaging filter; (2) aligning/interpolating the preprocessed measurement with the model reference response (inputs to the model-reference processor); (3) designing both optimal and adaptive processors, and (4) calculating the overall processing gain. It should be noted 


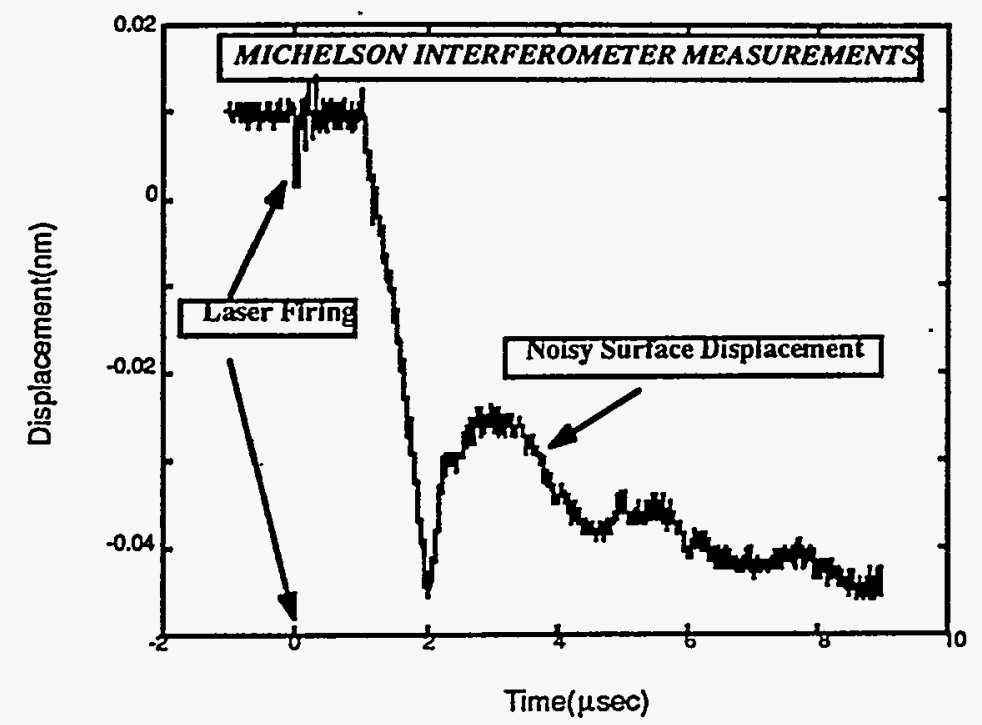

Figure 4: Typical Noisy Michelson Interferometer Experimental Measurement in $6 \mathrm{~mm}$ Aluminum Plate (30dB SNR).

that there are two caveats that can limit the performance of the model-reference processor: (i) the reference response is assumed to align temporally with the measurement; and (ii) the required parameters for the reference synthesis are known a-priori. Also note that in designing both processors, various filter orders, $(L)$, and regularization factors, $\sigma_{n}$ were selected during the design procedure. For each run the mean-squared error is calculated, and the set that yields the smallest error is chosen. A typical set is, $L=32$ with regularization factor, $\sigma_{n}=1 \times 10^{-3}$ yielding a mean-squared error on the order of $10^{-6}$.

An example SNR calculation run is shown in Figure 5 which shows the noisy measurement data, the model-reference signal, estimated noise (mean removal) and the enhanced signal estimate with associated SNR. Typically, the processing gain is $20 d B$ or greater. Figure 3 showed the results for processing a low SNR case while a high SNR case is shown below in Figure 6 which corresponds to the measurement in Figure 5. This figure shows the raw measurement data $\left(x\left(t_{k}\right)\right)$ and the optimal (upper trace) and adaptive (lower trace) processor surface displacement estimates $\left(\hat{d}\left(t_{k}\right)\right)$ overlayed on the interpolated model reference input $\left(d\left(t_{k}\right)\right)$. From these runs it is clear that the model-reference processor is capable of increasing the overall sensitivity ( $>20 d B$ ) of the Michelson interferometer measurements. This statement is further substantiated by calculating the SNRs (as above) for eleven experimental data sets on the aluminum plate (see Table I.).

\section{SUMMARY}

The sensitivity of a Michelson interferometric system to laser generated ultrasonic signals can be enhanced significantly using a model-reference processing approach. A model-reference response for a given specimen may be obtained using a sophisticated propagation model to predict the surface displacement resulting from laser generated ultrasound. Once generated, the reference is used to construct both the optimal and adaptive versions of the processor for potential on-line implementation. Greater than $20 \mathrm{~dB}$ gain in output SNR or equivalently, overall sensitivity improvement in the interferometric measurements was achieved using this approach.

The application of this model-reference approach requires the development of a reference 

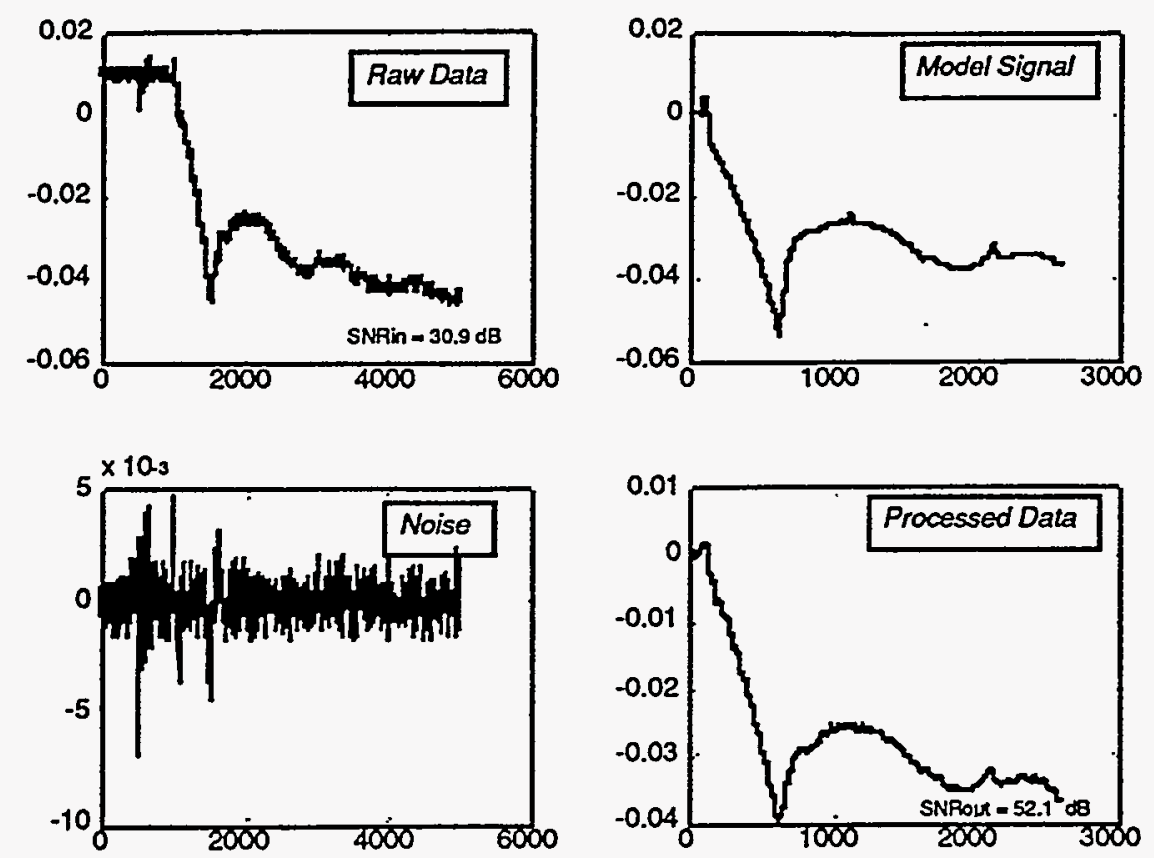

Figure 5: SNR Estimation: (a) Noisy measurement (30.9dB SNR). (b) Predicted Model-Reference Response. (c) Estimated Noise. (d) Optimal Surface Displacement Estimate(52.1dB SNR).

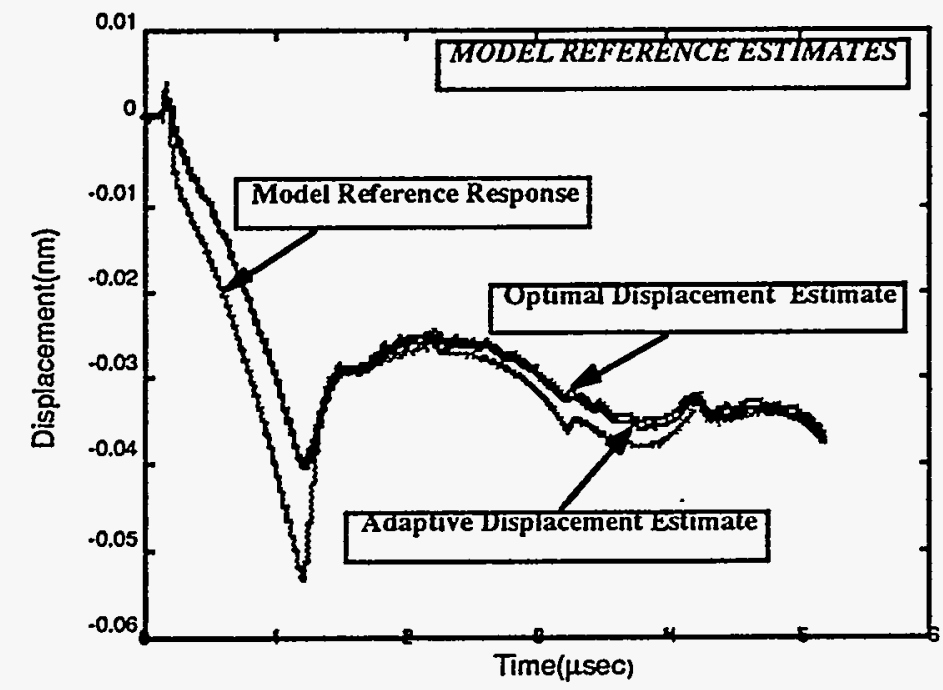

Figure 6: Enhanced Surface Displacement Estimate: Model Reference, Estimate, and Adaptive Estimate (30dB SNR).

Table I. Model-Reference Processsing: SNR Gains $(d B)$

\begin{tabular}{||c|c|c|c||}
\hline \hline Run No. & $S N R_{\text {in }}$ & $S N R_{\text {out }}$ & $S N R_{\text {gain }}$ \\
\hline 1 & 28.2 & 50.3 & 22.1 \\
\hline 2 & 28.5 & 50.1 & 21.6 \\
\hline 3 & 27.0 & 48.9 & 21.9 \\
\hline 4 & 27.8 & 49.7 & 21.9 \\
\hline 5 & 25.3 & 49.8 & 24.6 \\
\hline 6 & 30.9 & 52.1 & 21.2 \\
\hline 7 & 23.12 & 50.4 & 27.3 \\
\hline 8 & 25.4 & 52.0 & 26.6 \\
\hline 9 & 21.1 & 47.6 & 26.5 \\
\hline 10 & 22.9 & 49.4 & 26.5 \\
\hline 11 & 23.8 & 51.6 & 27.8 \\
\hline
\end{tabular}


response based on the underlying physics of the process under investigation, in this case, thermoelastic generation of ultrasound. To achieve a reasonable reference, the specimen and experimental parameters are required a-priori, and once developed the reference must be aligned with the measured signal or performance of the algorithm could be limited significantly. That is, performance can deteriorate significantly if the reference is not aligned properly with the measurement displacement. For this work, the alignment was accomplished interactively. An optimal approach that searches through all of the data to find the "best" alignment uses the so-called Simpson sideways recursion (see Robinson [4] for details) and the LWR algorithm discussed in Section II.

\section{ACKNOWLEDGEMENTS}

This work was performed by the Lawrence Livermore National Laboratory under the auspices of the U. S. Department of Energy, DOE Contract W-7405-ENG-48.

\section{REFERENCES}

1. J. B. Spicer and J. W. Wagner, "Comprehensive modelling of laser ultrasonic waveforms for materials characterization," in Acousto-Optics and Acoustic Microscopy, S. M. Gracewski and T. Kundu, eds., ASME AMD, 140 (American Society of Mechanical Engineers), 163-179, 1992.

2. J. D. Aussel and J. P. Monchalin, "Precision laser-ultrasonic velocity measurement and elastic constant determination," Ultrasonics, 27, 165-177, 1989.

3. J. V. Candy, Signal Processing: The Modern Approach. New York:McGraw-Hill, 1988.

4. E. Robinson and S. Treitel, Geophysical Signal Analysis. New Jersey:Prentice-Hall, 1980. 